\title{
Evidencia de sobreanticoagulación por warfarina en un hospital de segundo nivel de complejidad de Itagüí, Antioquia
}

1 Médico egresado Facultad de Ciencias de la Salud, Corporación Universitaria Remington, calle 51 \# 51-27, Medellín, Colombia. Correo: lauristobon@hotmail.com

2 Médico egresado Facultad de Ciencias de la Salud, Corporación Universitaria Remington, calle 51 \# 51-27, Medellín, Colombia. Correo: yulianamartinez67@hotmail.com

3 Magíster en Biología, integrante del Grupo de Investigaciones Biomédicas Uniremington, docente investigador Facultad de Ciencias de la Salud, Corporación Universitaria Remington, calle 51 \# 51-27, Medellín, Colombia. Correo: raul.rocha@uniremington.edu.co

4 Médica y cirujana, especialista en Toxicología Clínica, docente Facultad de Ciencias de la Salud, Corporación Universitaria Remington, calle 51 \# 51-27, Medellín, Colombia. Correo: isaescobartoledo@gmail.com

\author{
Evidence of warfarin-induced \\ over-anticoagulation in a secondary-care \\ hospital in Itagüí, Antioquia
}

Laura Restrepo Tobón'; Yuliana A. Giraldo Martínez²

Tutores: Raúl L. Rocha Orjuela3; Isabel E. Escobar Toledo ${ }^{4}$

\section{Resumen}

El objetivo de este estudio fue determinar la prevalencia y características generales de pacientes que presentaron sobreanticoagulación por warfarina durante el año 2011 en un hospital de segundo nivel de complejidad en Itagüí, Antioquia. Se realizó un estudio descriptivo retrospectivo durante el periodo comprendido entre el 1 de enero y el 31 de diciembre de 2011, considerando las historias clínicas de 203 pacientes admitidos al hospital y que fueron tratados de manera ambulatoria o intrahospitalaria con warfarina con el posterior evento adverso de sobreanticoagulación. La prevalencia de sobreanticoagulación con warfarina fue del $73 \%$. Se encontró un predominio del sexo femenino (60.70\%) con respecto al sexo masculino (38.10\%). El grupo etario más frecuente fue 70-79 años. La hipertensión arterial fue el antecedente más frecuente con (66.30\%), seguido por la fibrilación auricular (36.10\%) y la insuficiencia cardíaca congestiva (25.30\%). Los medicamentos asociados con evidencia de interacción farmacológica por tratamiento con warfarina fueron Furosemida (67.5\%), Omeprazol (45.8\%) y Enoxaparina (44.6\%). Por el contrario, los medicamentos Levotiroxina (15.7\%) y Amiodarona (20.5\%) se asociaron con ausencia de interacción farmacológica por el tratamiento anticoagulante. Según los resultados se hace necesario generar un hábito de prevención y cuidado sobre el uso de warfarina, un adecuado control y medición de la interacción con otros medicamentos.

Palabras clave: coagulación, reacción adversa, pacientes. 


\section{Abstract}

This study aims to determine the prevalence and general characteristics of patients who had warfarin-induced over-anticoagulation in 2011 at a secondary-care hospital in Itagüí (Antioquia). A retrospective descriptive study was conducted over the period between January 1 and December 31,2011 , considering the medical records of 203 patients admitted to the hospital and treated with warfarin, either as outpatients or inpatients, who had a subsequent adverse over-anticoagulation event. The prevalence of over-anticoagulation with warfarin was $73 \%$. A predominance of females $(60.70 \%)$ compared to males $(38.10 \%)$ was found. The most frequently affected age group was 70-79 years. Hypertension was the most common antecedent $(66.30 \%)$, followed by atrial fibrillation (36.10\%) and congestive heart failure (25.30\%). Medications associated with evidence of drug interaction, combined with warfarin, were Furosemide (67.5\%), Omeprazole (45.8\%) and Enoxaparin (44.6\%). By contrast, Levothyroxine (15.7\%) and Amiodarone (20.5\%) were not found to have a drug interaction with anticoagulant therapy. The results suggest the need to create a habit of prevention and care regarding the use of warfarin and to properly monitor and measure drug interactions.

Keywords: coagulation, adverse reaction, patients.

\section{Introducción}

Uno de los fármacos que inhibe los factores de coagulación dependientes de vitamina $K$ (Factores II, VII, IX y X y las proteínas anticoagulantes $\mathrm{C}$ y $S$ ) es la warfarina. Se ha indicado que este anticoagulante puede alterar los factores de coagulación indicados de manera dependiente de la dosis, o también con relación a otros fármacos, alimentos u otros (Bonilla et al. 2011).

Se ha descrito que la sobreanticoagulación con warfarina, causa una reacción adversa medicamentosa (RAM) que se mide de acuerdo con los tiempos de coagulación expresados en términos de INR (International Normalized Ratio), un valor mayor a 4 en esta escala define una sobreanticoagulación (Pineda, 2009). Dado que el uso de anticoagulantes orales de larga duración como la warfarina se ha incrementado, es necesario que el personal de las instituciones médicas esté atento a las reacciones que se puedan presentar y, más aún, por el hecho de interaccionar con otros medicamentos, sobre todo en personas de edad avanzada (Schulman, 2003; Jacobs, 2008; Wysowski et al., 2007; Mallet et al., 2007).

La carencia de clínicas especializadas en anticoagulación en la gran mayoría de instituciones prestadoras de servicios de salud en Colombia, hace que no existan directrices claras sobre el manejo y el seguimiento de estos pacientes. Muchos de los pacientes anticoagulados tienen una pobre red de apoyo social y son dependientes de un cuidador, lo que los hace más vulnerables. Además del riesgo trombótico existe el riesgo hemorrágico en estos pacientes facilitado por la polifarmacia, aumento del riesgo de caídas, trastornos demenciales, errores en los reajustes de la medicación y trastornos funcionales hepáticos de base.

Dado el uso de warfarina y el desconocimiento sobre las características en los pacientes y eventos de sobreanticoagulación, ligado a la interacción con otros medicamentos, se plantea como objetivo determinar la preva- 
lencia y características generales de pacientes que presentaron sobreanticoagulación por warfarina durante el año 2011 en un hospital de segundo nivel de complejidad en Itagüí, Antioquia.

\section{Materiales y métodos}

Se realizó un estudio descriptivo retrospectivo durante el periodo comprendido entre el 1 de enero y el 31 de diciembre de 2011 en pacientes que ingresaron al hospital y que fueron tratados de manera ambulatoria o intrahospitalaria con warfarina, con el posterior evento adverso de sobreanticoagulación. Se realizó un análisis de 203 historias clínicas, las cuales fueron facilitadas por el archivo del hospital. De este total 83 historias cumplieron los criterios de inclusión considerados para el estudio. Se incluyeron pacientes tratados exclusivamente con warfarina y con un tiempo de protrombina (TP) e International Normalized Ratio (INR) elevado ( $>4$ ) durante el año 2011. Aquellos pacientes con falla hepática prolongada, trastornos hemorrágicos de base o historia clínica incompleta se excluyeron del estudio.

La unidad de análisis fue la historia clínica de los pacientes. Adicionalmente, se utilizó la base de datos del Programa de Farmacovigilancia institucional para complementar los datos de los pacientes. Por ser un estudio de tipo retrospectivo, descriptivo, sin intervención, no fue necesario el consentimiento informado de los pacientes, pues se conservó el derecho a la privacidad de la historia clínica, y se contó con la aprobación del Comité de Ética del hospital.

\section{Análisis estadístico}

Las variables analizadas en el estudio comprendieron: sexo, edad, comorbilidades e interacción con otros medicamentos. Se realizó un análisis estadístico descriptivo básico de frecuencia de cada variable con el fin de evidenciar la reacción adversa al medicamento.

\section{Resultados}

Se revisaron 203 historias clínicas, las cuales fueron facilitadas por el archivo del hospital. De este total 83 historias cumplieron los criterios de inclusión considerados para el estudio. Según la información de las historias de la población de estudio $(n=83), 60.70 \%$ eran mujeres y $38.10 \%$ eran hombres.

La media de la edad fue 77.2 años, con una edad mínima de 36 años y una edad máxima de 91 años. Las edades se agruparon en rangos para su análisis como se puede apreciar en la tabla 1.

Tabla 1. Rangos de edad de la población de estudio.

\begin{tabular}{|c|c|}
\hline Rango de edad & Número de pacientes \\
\hline $30-39$ & 3 \\
\hline $40-49$ & 3 \\
\hline $50-59$ & 6 \\
\hline $60-69$ & 7 \\
\hline $70-79$ & 10 \\
\hline $80-89$ & 9 \\
\hline $90-99$ & 1 \\
\hline
\end{tabular}

Fuente. Elaboración propia.

Las patologías previas y su frecuencia registradas a partir de las historias clínicas revisadas se observa en la tabla 2. 
Tabla 2. Frecuencias de patologías y antecedentes de la población de estudio según la información registrada en las historias clínicas.

\begin{tabular}{|l|c|c|}
\hline \multicolumn{1}{|c|}{ Patología } & $\begin{array}{c}\text { Número de } \\
\text { hallazgos } \\
\text { del total }\end{array}$ & $\begin{array}{c}\text { Porcentaje } \\
(\%)\end{array}$ \\
\hline Hipertensión arterial & $55 / 83$ & 66.30 \\
\hline Fibrilación auricular & $30 / 83$ & 36.10 \\
\hline $\begin{array}{l}\text { Insuficiencia cardíaca } \\
\text { congestiva }\end{array}$ & $21 / 83$ & 25.30 \\
\hline $\begin{array}{l}\text { Enfermedad pulmonar } \\
\text { obstructiva crónica }\end{array}$ & $28 / 83$ & 33.70 \\
\hline $\begin{array}{l}\text { Trombosis profunda venosa } \\
\text { profunda }\end{array}$ & $15 / 83$ & 18.10 \\
\hline Tromboembolismo pulmonar & $12 / 83$ & 14.50 \\
\hline Diabetes mellitus & $11 / 83$ & 13.30 \\
\hline Insuficiencia renal crónica & $9 / 83$ & 10.80 \\
\hline Dislipemia & $9 / 83$ & 10.80 \\
\hline Recambio valvular & $7 / 83$ & 8.40 \\
\hline Evento cerebro vascular & $5 / 83$ & 6.00 \\
\hline Alzheimer & $2 / 83$ & 2.40 \\
\hline Arritmias & $2 / 83$ & 2.40 \\
\hline Hipertensión arterial pulmonar & $1 / 83$ & 1.20 \\
\hline Depresión & $1 / 83$ & 1.20 \\
\hline Hemartrosis & $1 / 83$ & 1.20 \\
\hline Hepatopatia & $1 / 83$ & 1.20 \\
\hline Esquizofrenia & $1 / 83$ & 1.20 \\
\hline Osteoartrosis & $1 / 83$ & 1.20 \\
\hline Artritis séptica & 1.20 \\
\hline Fuente. Elas & \\
\hline
\end{tabular}

Fuente. Elaboración propia.

La prevalencia de pacientes sobreanticoagulados con warfarina encontrada fue del $73 \%$ $(n=61)$. El $27 \%(n=22)$ presentaron valores normales de INR $(<4)$ como se aprecia en la figura 1.

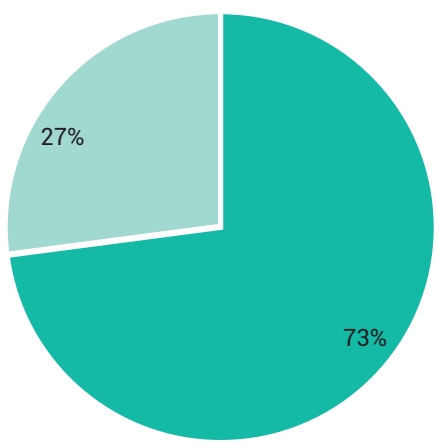

Sobreanticogulados No sobreanticogulados

Figura 1. Prevalencia de pacientes sobreanticogulados $y$ no sobreanticoagulados a partir de las historias clínicas revisadas. $\mathrm{n}=83$.

Fuente. Elaboración propia.

El estado de anticoagulación de los pacientes se obtuvo de los datos de la historia clínica. Los criterios definidos para determinar esta condición fueron el TP y el INR. Los valores TP se analizaron en tres tiempos distintos de la estancia del paciente: al inicio, en la estancia intermedia y al final (Figura 2).

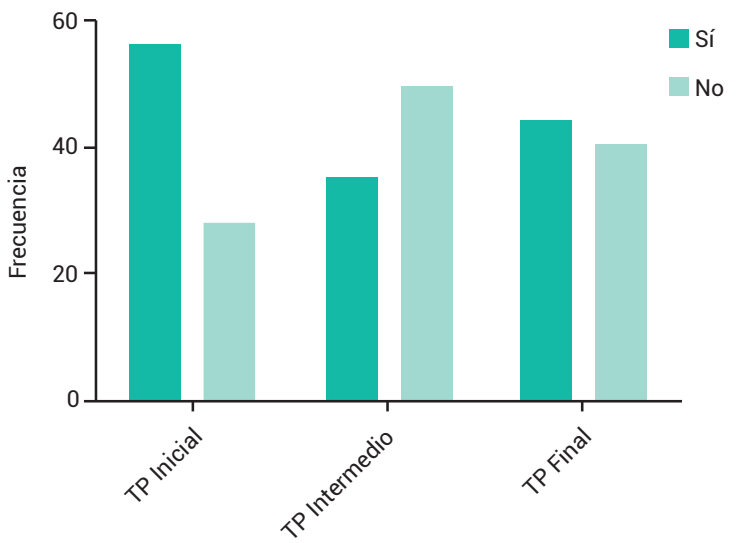

Figura 2. Descripción de la frecuencia de TP en los tiempos de evaluación determinados en el estudio.

Fuente. Elaboración propia. 
La segunda medida correspondiente al INR en tres tiempos durante la estancia en el hospital (Figura 3).

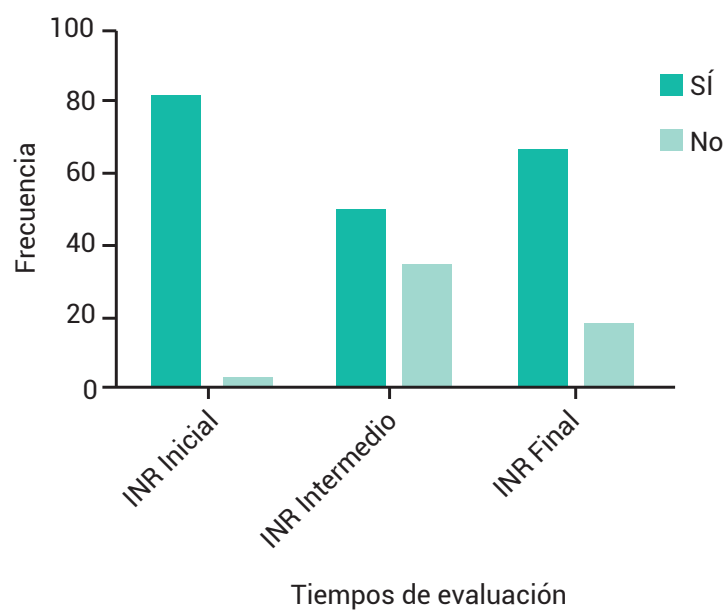

Figura 3. Descripción de la frecuencia de INR en los tiempos de evaluación determinados en el estudio.

Fuente. Elaboración propia.

Los medicamentos que presentaron mayor frecuencia y, además, asociados con el aumento del efecto farmacológico de la warfarina se indican en la tabla 3.

Tabla 3. Proporción de medicamentos combinados con el uso de warfarina asociados al aumento del efecto farmacológico.

\begin{tabular}{|l|c|c|}
\hline Medicamento & $\begin{array}{c}\text { Número de hallazgos } \\
\text { en historias clínicas }\end{array}$ & $\begin{array}{c}\text { Porcentaje de } \\
\text { frecuencia (\%) }\end{array}$ \\
\hline Furosemida & 56 & 67.5 \\
\hline Omeprazol & 38 & 45.8 \\
\hline Enoxaparina & 37 & 44.6 \\
\hline Lovastatina & 32 & 38.6 \\
\hline Acetaminofén & 31 & 37.3 \\
\hline Dipirona & 25 & 30.1 \\
\hline Enalapril & 23 & 27.7 \\
\hline Verapamilo & 23 & 27.7 \\
\hline Penicilina & 20 & 24.1 \\
\hline B. Ipratropio & 19 & 22.9 \\
\hline Salbutamol & 15 & 18.1 \\
\hline Digoxina & 12 & 14.5 \\
\hline
\end{tabular}

Fuente. Elaboración propia.
Otros medicamentos como nifedipino, captopril, metoclopramida, macrólidos, nadroparina, hidroclorotiazida, bisacodilo, haloperidol y bisacodilo, entre otros, fueron menos frecuentes en los hallazgos (datos no mostrados).

En la tabla 4 se muestran los medicamentos que presentaron mayor frecuencia y, además, asociados con la disminución del efecto farmacológico.

Tabla 4. Proporción de medicamentos combinados con el uso de warfarina asociados con la disminución del efecto farmacológico.

\begin{tabular}{|l|c|c|}
\hline Medicamento & $\begin{array}{c}\text { Número de hallazgos } \\
\text { en historias clínicas }\end{array}$ & $\begin{array}{c}\text { Porcentaje de } \\
\text { frecuencia (\%) }\end{array}$ \\
\hline Levotiroxina & 13 & 15.7 \\
\hline Amiodarona & 17 & 20.5 \\
\hline Amitriptilina & 2 & 2.4 \\
\hline Asa & 10 & 12 \\
\hline Carbamazepina & 1 & 1.2 \\
\hline Cefalosporinas & 5 & 6 \\
\hline Espironolactona & 26 & 31.3 \\
\hline Fenitoina & 2 & 2.4 \\
\hline Vitamina C & 3 & 3.6 \\
\hline Vitamina K & 42 & 50.6 \\
\hline Sucralfate & 7 & 8.4 \\
\hline Trazodona & 5 & 6 \\
\hline
\end{tabular}

Fuente. Elaboración propia.

\section{Discusión}

Nuestro estudio aporta resultados sobre el uso de warfarina en pacientes tratados de manera ambulatoria o intrahospitalaria en un hospital de segundo nivel de complejidad de Itagüí, Antioquia, que presentaron como efecto adverso sobreanticoagulación. Nuestros hallazgos relacionan variables importantes como el sexo, grupo etario, comorbilidades e interacciones 
medicamentosas cuando se usa warfarina como tratamiento y se llama la atención sobre su prevención.

Un hallazgo importante es la gran prevalencia de sobreanticoagulación con warfarina que arrojó un valor del $73 \%$ de los pacientes que cumplieron los criterios de inclusión, evidenciando un alto índice de la misma. Este resultado es similar a lo reportado en estudios previos con warfarina en nuestro país, donde se indica un $72 \%$ de pacientes sobreanticoagulados (Pineda, 2009). Este dato aporta a los estudios sobre los efectos adversos de la warfarina, que en nuestro país son escasos.

Respecto a las características de la población encontramos un predominio del sexo femenino, lo cual concuerda con otros estudios, posiblemente debido a que este género es el que más consulta el servicio de urgencias, además de consumir de una manera más estricta la medicación ordenada por el médico, lo que puede conllevar a situaciones de polimedicación, aumentando el efecto de warfarina en muchos casos (Pineda, 2009).

Los resultados indican una tendencia en cuanto a grupo etario, donde la población más afectada se compone de pacientes de la tercera edad, principalmente en mayores de 70 años. Esto se debe a la asociación con múltiples comorbilidades, polimedicación y características metabólicas que les confieren más riesgo (Quintero-González, 2010; Yurgaky y Rodríguez, 2009; Ramírez Martínez, 2010; Souto y Ruyra, 2010; Quintero-González, 2010).

Encontramos, además, que los pacientes con edad superior a 50 años conformaban más de la mitad de la población, hecho que coincide con lo que han reportado otros autores (Pineda, 2009; Quintero-González, 2010). La edad es un factor de riesgo para efectos adversos a medicamentos y más aún a la sobreanticoagulación por warfarina, es por esta razón que este grupo poblacional visita con mayor frecuencia los servicios de urgencias.

Las múltiples comorbilidades de los pacientes que tienen tratamiento de manera crónica han sido analizadas en múltiples estudios a nivel local e internacional (Quintero-González, 2010; Yurgaky y Rodríguez, 2009; Souto y Ruyra, 2010; Zarzuelo Zurita y Morón Romero, 2009; Mendoza, 2005). En nuestra investigación se encontró que la gran mayoría de pacientes tenía otras patologías o antecedentes de ellas. Es un hecho frecuente la presencia de pacientes que sufren alguna enfermedad y que deben consumir medicación incluso de forma crónica. Esta situación incrementa el riesgo de interacciones o de errores en su posología como lo evidencian estudios previos en nuestro país (Ramírez Martínez, 2010). Según nuestros hallazgos la hipertensión arterial fue el antecedente más frecuente, seguido por la fibrilación auricular y la insuficiencia cardíaca congestiva.

Aunque no se determinó el número de medicamentos por paciente, sus antecedentes permiten inferir que se da el consumo de múltiples fármacos y las patologías más comunes requieren polifarmacia para su adecuado control (Pineda, 2009; Quintero-González, 2010; Yurgaky y Rodríguez, 2009; Ramírez Martínez, 2010; Souto y Ruyra, 2010; Mendoza, 2005; Mancera Madrigal y Azyadet Parra, 2008).

Un sesgo de nuestro estudio fue la ausencia de datos de TP e INR en un gran porcentaje de pacientes en la fase media de estancia y al final, lo cual no pudo ser controlado. Este hecho es de gran importancia como lo demuestra un estudio realizado en 1999 en Venezuela, donde se analizó de manera retrospectiva (50 
años) el uso de la warfarina. En este estudio se resalta en gran medida el valor del seguimiento con TP e INR, debido a la gran cualidad de la warfarina para prolongar el tiempo de protrombina (Quintero-González, 2010). Otra falencia de nuestro estudio fue la falta de información en algunos aspectos aportados por los formatos de farmacovigilancia, que impidieron una mejor generación de datos, como el caso del poco seguimiento del TP e INR en algunos pacientes.

En nuestro estudio hubo una importante recolección de datos sobre medicamentos de uso en conjunto con la warfarina y se profundizó en cuáles aumentaba o disminuía el efecto de la misma, encontrando asociación de la polimedicación con la sobreanticoagulación. Este resultado concuerda con resultados anteriores donde ciertos medicamentos se asociaron más con sobreanticoagulación al ser usados en conjunto con warfarina (Pineda, 2009; Holbrook et al., 2005).

Pese a que el estudio presentado no pudo profundizar acerca de las correlaciones del efecto de sobreanticoagulación con todas las variables, los resultados nos informan y actualizan con respecto a la sobreanticoagulación con warfarina en pacientes que lo consumen tanto de forma ambulatoria como intrahospitalaria en un hospital de segundo nivel de complejidad del municipio de Itagüí, Antioquia. A la luz de nuestros resultados se hace necesario generar un hábito de prevención y cuidado sobre el uso de la warfarina, su adecuado control y medición y el uso de medicamentos asociados. De acuerdo con este hecho se recomendaría un mayor control en la dosificación del medicamento y realizar controles seriados con medición de tiempos de coagulación, acortando los periodos de intermedición para evitar culminar en este evento adverso.

\section{Agradecimientos}

Los autores agradecen al personal de la E.S.E Hospital San Rafael de Itagüí por su colaboración para el desarrollo del estudio. A la Facultad de Ciencias de la Salud de la Corporación Universitaria Remington por su apoyo para el desarrollo de este proyecto y por propiciar espacios para la investigación.

\section{Referencias}

- Bonilla Hernández, J. D., Leal Cardoso, M. A., López Artunduaga, L. T., Poveda Conde, L. C., y Caviedes Pérez, G. (2011). Prevalencia y factores de riesgo relacionados con sangrado en la terapia con warfarina de los pacientes que ingresan al HUHMP de Neiva en el año 2011. Revista Facultad de Salud RFS, 3(2), 23-31.

- Holbrook, A., Pereira, J., Labiris, R., McDonald, H., Douketis, J., Crowther, M., y Wells, P. (2005). Systematic overview of warfarin and its drug and food interactions. Archives of internal medicine, 165(10), 1095-1106. Recuperado de http://archinte.jamanetwork. com/article. aspx?articleid $=486574$

Jacobs, L. G. (2008). Warfarin pharmacology, clinical management, and evaluation of Hemorrhagic Risk for the Elderly. Cardiology Clinics, 26(2), 157-167. Recuperado de http://www.cardiology.theclinics.com/article/S0733-8651(07)00148-8/abstract

- Mallet, L., Spinewine, A., y Huang, A. (2007). The challenge of managing drug interactions in elderly people. Lancet, 14(370), 185191. Recuperado de http://www.thelancet. 
com/journals/lancet/article/PIIS01406736(07)61092-7/abstract

- Mancera Madrigal, C. S., y Azyadet Parra, B. (2008). Anticoagulación vía oral. Revista Mexicana de Enfermería Cardiológica, 16(1), 11-19. Recuperado de http://www.medigraphic.com/pdfs/enfe/en-2008/en081c.pdf

- Mendoza, B. (2005). Manejo de la sobreanticoagulación y el sangrado en pacientes anticoagulados. En O. Gómez, Anticoagulación (pp. 83-88). Bogotá: Sociedad Colombiana de Cardiología.

- Pineda, J. (2009). Sobreanticoagulación con warfarina. Archivos de Medicina, 9(2), 174-182.

- Quintero-González, J. A. (2010). Cincuenta años de uso clínico de la warfarina. Invest clín, 51(2), 269-287. Recuperado de http://www.scielo.org.ve/scielo.php?pi$d=\$ 0535-51332010000200008$ \&script=sci_arttext

Ramírez Martínez, J. A. (2010). Estudio Colombiano para el diseño de un puntaje predictor de riesgo de sangrado entre los pacientes usuarios de Warfarina (Tesis de especialización en Medicina Interna). Bd digital Unal. Recuperado el 20 de Agosto de 2015 , de http://www.bdigital.unal.edu. co/2513/1/597924.2010.pdf
Schulman, S. (2003). Clinical practice care of patients receiving log term anticoagulant therapy. The New England journal of medicine, 349(7), 675-683. Recuperado de http://www. ncbi.nlm.nih.gov/pubmed/12917305

- Souto, J., y Ruyra, X. (1 de Noviembre de 2010). Monitor Medical. Recuperado de http://www.monitormedical.es/files/files/20110621165838_728031.pdf

- Wysowski, D. K., Nourjah, P., y Lynette, S. (2007). Bleeding complications with warfarin use: a prevalent adverse effect resulting in regulatory action. Archives of internal medicine, 9(167), 1414-1419. Recuperado de http://archinte.jamanetwork.com/article.aspx?articleid $=412786$

- Yurgaky, J., y Rodríguez, F. (2009). Warfarina: uso contemporáneo. Rev Fac Med, 17(1), 107-115. Recuperado de http://www.scielo. org.co/pdf/med/v17n1/v17n1a15

- Zarzuelo Zurita, A., y Morón Romero, R. (2009). Interacciones anticoagulantes orales (I). Generalidades de la trombosis y aspectos farmacológicos de los anticoagulantes orales. Aula de la farmacia: revista profesional de formación continuada, 5(59), 46-54. Recuperado de http:// dialnet.unirioja.es/servlet/articulo?codigo $=4693078$ 